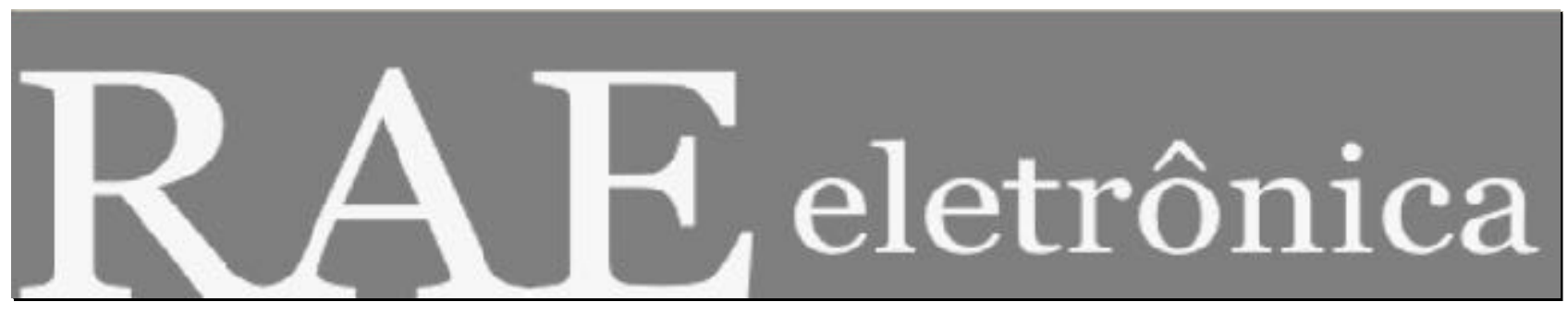

\title{
A CONFIANÇA NOS RELACIONAMENTOS INTERORGANIZACIONAIS: O CAMPO DA BIOTECNOLOGIA EM ANÁLISE
}

Por:

\section{Cleverson Renan da Cunha, UNIVERSIDADE FEDERAL DO PARANÁ Marlene Catarina de Oliveira Lopes Melo, UNIHORIZONTES}

RAE-eletrônica, v. 5, n. 2, Art. 18, jul./dez. 2006

http://www.rae.com.br/eletronica/index.cfm?FuseAction=Artigo $\& I D=3689 \&$ Secao=ARTIGOS $\&$ Volu $\mathrm{me}=5 \&$ Numero $=2 \& \mathrm{Ano}=2006$

CCopyright, 2006, RAE-eletrônica. Todos os direitos, inclusive de tradução, são reservados. É permitido citar parte de artigos sem autorização prévia desde que seja identificada a fonte. A reprodução total de artigos é proibida. Os artigos só devem ser usados para uso pessoal e nãocomercial. Em caso de dúvidas, consulte a redação: raeredacao@fgvsp.br.

A RAE-eletrônica é a revista on- line da FGV-EAESP, totalmente aberta e criada com o objetivo de agilizar a veiculação de trabalhos inéditos. Lançada em janeiro de 2002, com perfil acadêmico, é dedicada a professores, pesquisadores e estudantes. Para mais informações consulte o site www.rae.com.br/eletronica.

\section{RAE-eletrônica}

\section{ISSN 1676-5648}

(C2006 Fundação Getulio Vargas - Escola de Administração

de Empresas de São Paulo.

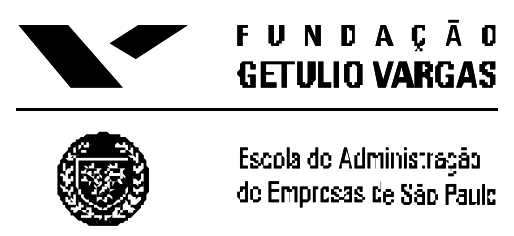




\section{A CONFIANÇA NOS RELACIONAMENTOS INTERORGANIZACIONAIS: \\ O CAMPO DA BIOTECNOLOGIA EM ANÁLISE \\ Cleverson Renan da Cunha - Marlene Catarina de Oliveira Lopes Melo}

\section{RESUMO}

A confiança tem sido destacada como um instrumento vital para a realização de parcerias mais flexíveis e eficientes, em detrimento de instrumentos de controle coercitivo. Isso é ainda mais evidente, em campos organizacionais que trabalham com inovação e conhecimento de fronteira, como na biotecnologia. Assim, buscourse analisar a dinâmica da confiança no processo de desenvolvimento dos relacionamentos interorganizacionais que visam à pesquisa e ao desenvolvimento de novos produtos e/ou serviços em empresas de biotecnologia de saúde humana no Brasil. A análise evidenciou a importância da confiança para a realização das parcerias, demonstrando a existência de dois domínios: das relações interorganizacionais e das relações interpessoais, com a preponderância do último em decorrência do baixo nível de institucionalização dos sistemas abstratos que fundamentam a confiança interorganizacional.

\section{PALAVRAS-CHAVE}

Relacionamento interorganizacional, confiança, biotecnologia, cooperação, desenvolvimento tecnológico.

\section{ABSTRACT}

Trust has been highlighted as a vital for the accomplishment of more flexible and efficient partnerships - rather than coercive control instruments - mainly in organizational fields, which deal with innovation and frontier knowledge, like biotechnology. The article analyses the dynamics of trust in the developmental process of interorganizational relationship directed at researching and developing new products and/or services in human health biotechnology in Brazilian companies. Analysis of the cases identified trust as vitally important for the establishment of partnerships opening up two domains: interorganizational and interpersonal relationships, being the latter as more important.

\section{KEYWORDS}

Interorganizational relationship, trust, biotechnology, cooperation, technological development. 


\section{A CONFIANÇA NOS RELACIONAMENTOS INTERORGANIZACIONAIS: \\ O CAMPO DA BIOTECNOLOGIA EM ANÁLISE \\ Cleverson Renan da Cunha - Marlene Catarina de Oliveira Lopes Melo}

\section{INTRODUÇÃO}

Os desafios enfrentados pelas organizações contemporâneas têm incentivado a formulação de alianças e parcerias entre organizações, nos contextos nacional e internacional, influenciando o desenvolvimento de pesquisas sobre o relacionamento entre organizações. Nesses temas, um dos que têm recebido atenção é o processo de desenvolvimento de pesquisas tecnológicas realizadas além dos limites organizacionais, principalmente os que tratam de organizações que faze m uso intensivo de conhecimento, como as empresas de biotecnologia (Powell, Koput e Smith-Doerr, 1996).

Nesse cenário a confiança é um dos mais importantes e freqüentes conceitos mencionados na conexão das relações cooperativas entre firmas (Grandori e Soda, 1995), principalmente quando estão envolvidos processos de aprendizagem e inovação (Dodgson, 1993). A existência da confiança entre parceiros na formação e manutenção das alianças pode reduzir os custos de coordenação e a necessidade de controles hierárquicos, o que tende a aumentar a flexibilidade da organização e a aumentar sua capacidade de se adequar às novas necessidades (Alter e Hage, 1993).

A importância da confiança é ainda maior se considerarmos as empresas que fazem uso intensivo de tecnologia e conhecimento, como as indústrias aeroespacial, de tecnologia de informação e de biotecnologia (Stuart, 1998). Aqui há necessidade crescente de trocas de informações e recursos entre as empresas em virtude da instabilidade inerente a esses negócios. No entanto, os estudos da confiança têm se concentrado nesse conceito como antecedente (Argyle, 1991) ou como resultante dos relacionamentos (Oliver, 1998). Ring e Van de Ven (1994) sugerem que, além dessas questões, o processo do desenvolvimento dos relacionamentos entre as organizações deve ser objeto de estudo. As fases de negociação, elaboração de compromissos, decisão e execução do relacionamento são decisivas para que se entenda o relacionamento de uma maneira mais ampla.

De outra forma, a biodiversidade presente no território brasileiro e os avanços alcançados em algumas áreas como genética animal e vacina humana têm colocado o Brasil em uma posição de relativo destaque no contexto mundial. Aliado a isso, a biotecnologia tem sido apontada como uma grande promessa para o desenvolvimento econômico nacional, evidenciando a importância de estudos nessa área. Sendo assim, optou-se por estudar os relacionamentos interorganizacionais cooperativos de empresas de biotecnologia que se dedicam à área de saúde humana no Brasil.

Entendendo que os relacionamentos interorganizacionais são influenciados por seu contexto, busca-se com o presente estudo analisar a confiança no relacionamento interorganizacional cooperativo 


\section{A CONFIANÇA NOS RELACIONAMENTOS INTERORGANIZACIONAIS: \\ O CAMPO DA BIOTECNOLOGIA EM ANÁLISE \\ Cleverson Renan da Cunha - Marlene Catarina de Oliveira Lopes Melo}

para pesquisa e desenvolvimento entre empresas do campo da biotecnologia, de uma perspectiva supraorganizacional e qualitativa, baseada em estudos múltiplos de caso.

\section{CAMPO DA BIOTECNOLOGIA NO BRASIL}

Desde que foi empregado pela primeira vez em 1919, o termo biotecnologia tem sido aplicado a várias situações e nos mais diferentes contextos. Uma análise etimológica da palavra remete ao grego, com a junção de bio (vida), logos (conhecimento) e tecnos (utilização prática). Tem-se que a biotecnologia é a aplicação do conhecimento prático relacionado à vida. No entanto, essa palavra tem sido empregada para classificar uma "indústria" ou um setor econômico, um campo de pesquisa ou a aplicação de determinadas técnicas.

Trigueiro (2002, p. 17) afirma que "a biotecnologia moderna, diferente da tradicional, emerge como trama complexa de relações técnicas, sociais, econômicas, políticas, éticas e institucionais, demandando um esforço transdisciplinar e interinstitucional para seu desenvolvimento". Para o autor, ela possui duas dimensões: a científica e a tecnológica. Na primeira, a nova biotecnologia consiste em um conjunto articulado de programas de pesquisas básicas (biologia molecular, bioquímica, microbiologia, genética), sendo desenvolvido, fundamentalmente, nas universidades e instituições acadêmicas. Na segunda, há a dificuldade de transformar os projetos de bancada em aplicações industriais e comerciais. Essas duas dimensões coexistem e se complementam. No Brasil, a primeira dimensão tem presenciado grandes avanços como o seqüenciamento genético da praga do amarelinho, que ataca os laranjais, ou o programa do genoma da cana. No entanto, o país ainda apresenta grande déficit quando se trata da aplicação industrial das descobertas e dos avanços obtidos nas pesquisas.

Uma outra diferenciação é feita por Carvalho (2004), que distingue as indústrias biotecnológicas em duas arenas. A primeira é formada pelas empresas que produzem inovação tecnológica pelo desenvolvimento de novos métodos, processos e produtos e/ou desenvolvem e produzem equipamento crítico e/ou componentes para a pesquisa e o desenvolvimento de biotecnologia. A segunda é formada pelas empresas que fazem uso de insumos e processos biotecnológicos nas suas linhas de produção de bens e serviços. As atividades necessárias a descobertas, produção, aprovação e comercialização dos produtos biotecnológicos normalmente envolvem altos custos financeiros e econômicos, seja para equipar os laboratórios, formar e manter recursos humanos, seja para custear os testes parametrizados e sistemáticos necessários, desde a 


\section{A CONFIANÇA NOS RELACIONAMENTOS INTERORGANIZACIONAIS: \\ O CAMPO DA BIOTECNOLOGIA EM ANÁLISE \\ Cleverson Renan da Cunha - Marlene Catarina de Oliveira Lopes Melo}

pesquisa até o lançamento do produto final. Por estarem lidando com tecnologias no limiar do conhecimento, apresentam altos custos relacionados com o trabalho científico original, assim como de avaliação da eficácia de seu desempenho.

Juntamente com o risco econômico, decorrente de investimentos em pesquisas que podem não trazer o retorno previsto, deve ser associado, igualmente, um risco social relacionado aos eventuais impactos provenientes da reação da sociedade diante dos produtos lançados comercialmente (Trigueiro, 2002). Esse segundo tipo de risco faz com que o setor seja altamente controlado tanto pela sociedade, por meio de organizações não-governamentais, como pelas agências do governo.

Neste artigo a biotecnologia será analisada em um contexto mais amplo, formado não apenas pelas técnicas, mas pelos diversos atores com ela envolvidos, desde os cientistas que se dedicam à ciência básica até os consumidores e as agências de regulamentação. Ela será usada como núcleo para a definição de um campo organizacional.

Para DiMaggio e Powell (1983), um campo organizacional se refere ao conjunto de organizações que constituem uma área reconhecida da vida organizacional. No caso da biotecnologia, envolve os pesquisadores, os fornecedores de equipamentos, as indústrias que fazem uso de suas técnicas, agências governamentais, organizações não-governamentais e grupos de consumidores, entre outros. O processo de estruturação de um campo organizacional envolve, ainda de acordo com os dois autores anteriormente citados, quatro fatores, quais sejam: aumento de interação entre as organizações do campo; emergência de dominações interorganizacionais e padrões de coalizão consistentemente definidos; aumento na carga de informações; e desenvolvimento de uma consciência mútua entre os atores de sua participação nesse campo e das atividades desenvolvidas em comum. Segundo Powell (1999), esses fatores ainda estão em processo de construção do campo analisado.

Dadas às características do campo da biotecnologia, a formação de relacionamentos interorganizacionais cooperativos tem sido adotada por diversas organizações, dos mais variados tamanhos e setores. Entender essas particularidades pode contribuir para o melhor entendimento dos mecanismos de elaboração e manutenção da confiança no seio das empresas e, conseqüentemente, facilitar os processos associativos, tão importantes nesse estágio de evolução do campo. 


\section{A CONFIANÇA NOS RELACIONAMENTOS INTERORGANIZACIONAIS: \\ O CAMPO DA BIOTECNOLOGIA EM ANÁLISE \\ Cleverson Renan da Cunha - Marlene Catarina de Oliveira Lopes Melo}

\section{RELACIONAMENTOS INTERORGANIZACIONAIS COOPERATIVOS}

As relações interorganizacionais se referem a qualquer tipo de contatos entre duas ou mais organizações, variando da forma concorrencial e antagônica para aquelas de natureza cooperativa, tanto entre organizações similares ou diferentes, envolvendo transações, fluxos e ligações de recursos relativamente duradouros (Oliver, 1990). A existência de contatos inter-relacionais implica que as organizações envolvidas desenvolvam processo de escolha do grupo de interação com aqueles que são relevantes no conjunto de organizações (Ring e Van de Ven, 1994). Entre esses relacionamentos, percebe-se um aumento da importância das relações entre empresas visando à colaboração como instrumento estratégico. Nesse sentido, diversos motivos são apresentados para a justificação da participação em um arranjo interorganizacional, forçados pela incapacidade das organizações de se auto-sustentarem (Aldrich, 1979). Para Aldrich as organizações também se esforçam para serem autônomas e, por opção, elas prefeririam não estabelecer relações interorganizacionais, na medida em que essas relações tendem a limitar suas ações subseqüentes, o que dificulta a escolha das estratégias.

A partir dos anos de 1980, as alianças vêm recebendo atenção considerável dos estudiosos, principalmente da economia, da sociologia e da teoria organizacional. A atenção tem se concentrado no estudo dos motivos que levam as empresas a formar parcerias (Oliver, 1998), nos tipos de relacionamentos (Oliver, 1990) e nos novos formatos organizacionais (Miles e Snow, 1992). Para Ring e Van de Ven (1994) pouca atenção tem sido devotada ao desenvolvimento das alianças, em detrimento das propriedades estruturais ou das condições antecedentes. Os relacionamentos interorganizacionais cooperativos devem ser vistos como mecanismos socialmente constituídos para a ação coletiva, sendo constantemente formados e reestruturados pelas ações e pelas interpretações simbólicas das partes envolvidas. A importância de se analisar as alianças como um processo é a de que os agentes das organizações precisam conhecer, além das condições de investimentos, os tipos de estrutura de governança requeridos para um relacionamento. A forma como se negociam, executam e modificam os termos de um relacionamento influencia fortemente o julgamento das partes, se as ações tomadas no relacionamento são eqüitativas e eficientes, além de influenciar na motivação para a continuação ou término do relacionamento (Ring e Van de Ven, 1994).

Nessa abordagem, os estágios de negociação, de compromisso e execução se dão em uma seqüência repetitiva e referenciada pela busca da eficiência e eqüidade (veja a Figura 1). Na fase da negociação, as partes desenvolvem expectativas em conjunto sobre suas motivações, possíveis investimentos e incertezas percebidas da parte do negócio que elas querem empreender conjuntamente. 


\section{A CONFIANÇA NOS RELACIONAMENTOS INTERORGANIZACIONAIS: \\ O CAMPO DA BIOTECNOLOGIA EM ANÁLISE \\ Cleverson Renan da Cunha - Marlene Catarina de Oliveira Lopes Melo}

Nesse momento o foco está no processo de barganha formal e no processo psicossocial de formação do senso comum.

FIGURA 1 - Arcabouço do desenvolvimento dos relacionamentos interorganizacionais cooperativos na abordagem de processos.

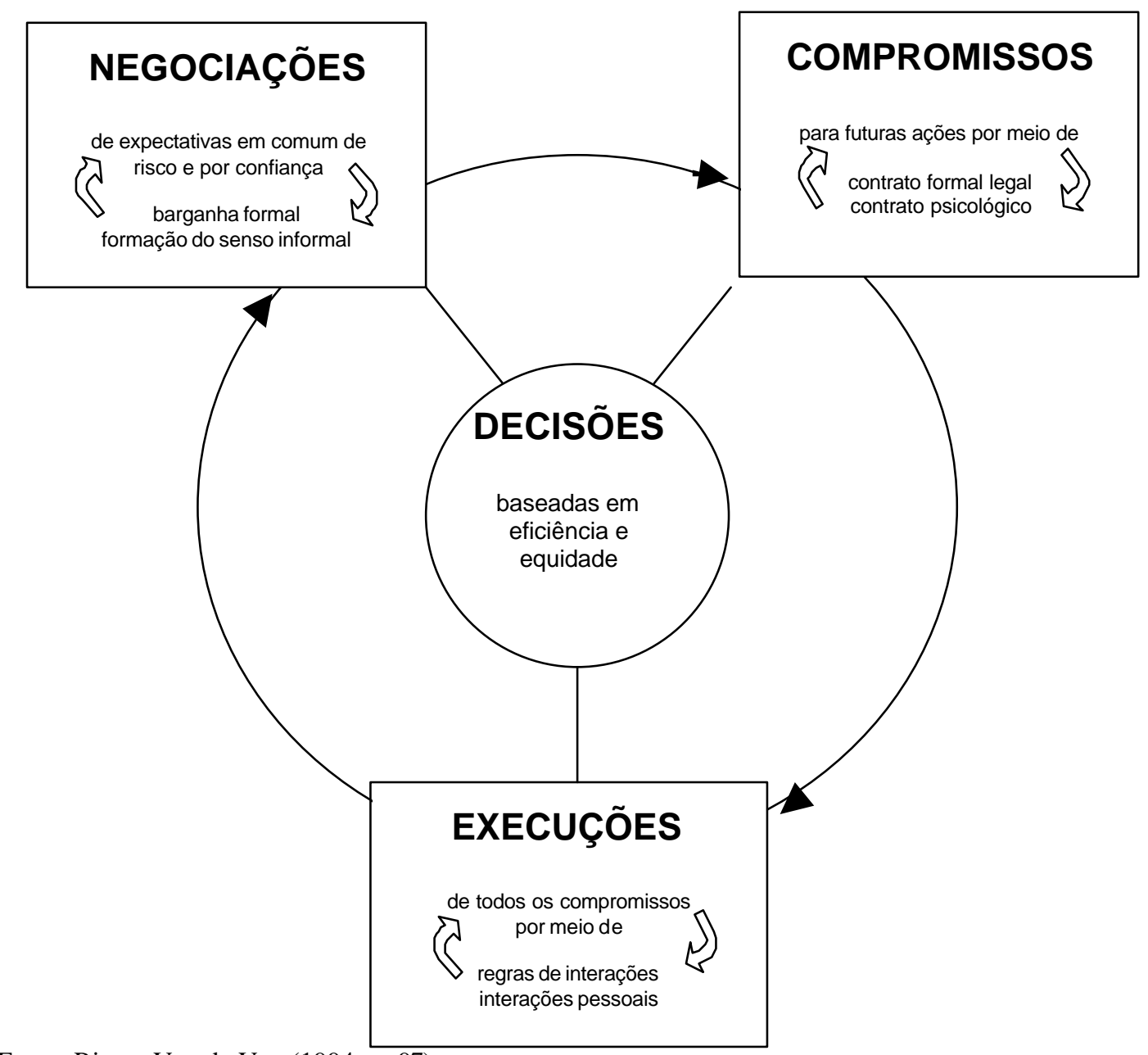

Fonte: Ring e Van de Ven (1994, p. 97).

No estágio de compromissos, são determinadas as obrigações e normas que orientarão os futuros relacionamentos. Nesse ponto, os termos e as estruturas de governança são estabelecidos, dependendo dos riscos a serem enfrentados. No estágio da execução, os compromissos e as regras de ação são implementados. As partes dão ordens a seus funcionários, compram materiais e se aplicam outros procedimentos administrativos. 


\section{A CONFIANÇA NOS RELACIONAMENTOS INTERORGANIZACIONAIS: \\ O CAMPO DA BIOTECNOLOGIA EM ANÁLISE \\ Cleverson Renan da Cunha - Marlene Catarina de Oliveira Lopes Melo}

Como não é possível estabelecer a priori todas as mudanças e as respectivas reações dos atores a elas, a incerteza está sempre presente nos relacionamentos. Os indivíduos procuram conciliar seus interesses com a necessidade de manter seus relacionamentos sociais.

Nos relacionamentos organizacionais, a plena conexão entre os parceiros raramente é realizada de uma única vez, pois é fruto da interação contínua, permitindo que cada membro da aliança compreenda os outros. À medida que o relacionamento se desenvolve, amplia-se também o nível de compreensão e de confiança, tornando-se mais confortável lidar com as incertezas que surgem na aliança. Portanto, para Doz e Hamel (2000), uma aliança talvez seja mais bem concebida como um relacionamento evolutivo, pontuado por uma série de comprometimentos, etapas e trocas negociadas explicitamente e aceitas de forma implícita ao longo do tempo. Nesse sentido, a colaboração acontece quando um parceiro demonstra ao outro confiança e utiliza oportunidades para se mostrar confiável, favorecendo inclusive o desenvolvimento de um ciclo de aprendizagem virtuoso.

Achrol, Scheer e Stern (1990) identificam várias questões referenciais para o entendimento das alianças entre empresas, destacando o comprometimento dos membros, a coesão do grupo e a motivação para participar da aliança. Acima de tudo, os autores destacam a confiança como fator crítico para o relacionamento entre parceiros, uma vez que investimentos de longo prazo em negócios e as possíveis mudanças que podem ocorrer não são especificados ou controlados pelas partes anteriormente (Ring e Van de Ven, 1994).

\section{CONFIANÇA NOS RELACIONAMENTOS COOPERATIVOS}

O estudo da confiança tem recebido grande atenção e reconhecida como um mecanismo cultural importante na condução das interações e expectativas de atores sociais (Fukuyama, 1995). Para Bachmann et al. (2001), sem um mínimo de confiança é quase impossível o estabelecimento e a manutenção de relações organizacionais bem-sucedidas por um longo período. Isto é ainda mais verdadeiro no contexto das rápidas mudanças presentes atualmente no mundo dos negócios e o fato de o controle ser tradicionalmente visto como mecanismo de coordenação pejorativo, particularmente por aqueles que a ele estão sujeitos.

Por ser um conceito multidimensional (Newell e Swan, 2000), a confiança pode apresentar vários significados e compartilha, segundo Lane (1998), três pressupostos: existência de um grau de interdependência entre o que confia e o que recebe a confiança; a confiança provê a forma de arcar com 


\section{A CONFIANÇA NOS RELACIONAMENTOS INTERORGANIZACIONAIS: \\ O CAMPO DA BIOTECNOLOGIA EM ANÁLISE \\ Cleverson Renan da Cunha - Marlene Catarina de Oliveira Lopes Melo}

riscos ou incertezas nos relacionamentos de troca; e a crença ou a expectativa de que a vulnerabilidade resultante da aceitação do risco não irá tirar vantagem do relacionamento.

A confiança também está relacionada com os processos de institucionalização de uma dada sociedade, como nos sugerem as idéias de Giddens (1991). Ele percebe que a alteração das bases da confiança na sociedade é uma evidência importante da mudança nos padrões de construção das relações sociais e de intimidade, evoluindo de bases pessoais para a confiança em sistemas abstratos. Para ele, não se trata da supressão das relações pessoais, mas de sua transformação, sendo agora intencionalmente construídas. Uma das fundamentações dessa confiança está nos sistemas peritos, como os mecanismos de governança, na Bolsa de Valores e no sistema monetário, entre outros, que fornecem garantias.

No Quadro 1 é apresentada uma tentativa de evidenciar as diferenças do ambiente da confiança entre padrões racionalizados da vida moderna e outros de base mais tradicional. Cada uma tende a variar segundo a ordem social específica em questão.

\section{QUADRO 1 - Ambientes de confiança nas culturas pré-modernas e modernas.}

\section{PRÉ-MODERNAS}

\section{Contexto geral: importância excessiva na confiança} localizada

1. Relações de parentesco como um dispositivo de organização para estabilizar laços sociais através do tempoespaço;

2. Comunidade local como um lugar, fornecendo um meio familiar;

3. Cosmologias religiosas como modos de crença e práticas rituais fornecendo uma interpretação providencial da vida humana e da natureza;

4. Tradição como um meio de conectar presente e futuro; orientada para o passado em tempo reversível.

\section{MODERNAS}

Contexto geral: relações de confiança em sistemas abstratos desencaixados

1. Relações pessoais de amizade ou intimidade sexual como meio de estabilizar laços sociais;

2. Sistemas abstratos como meios de estabilizar relações através de extensões indefinidas de tempo-espaço;

3. Pensamento contrafatual orientado para o futuro como um modo de conectar passado e presente.

Fonte: Adaptado de Giddens (1991, p. 104).

Ao analisar o trabalho de Giddens, percebem-se três grandes forças dinâmicas na sociedade contemporânea: a separação do tempo e espaço; a existência de relações sociais em contextos sociais locais, sem que necessariamente haja contato face a face, mediadas pela confiança em meios de 


\section{A CONFIANÇA NOS RELACIONAMENTOS INTERORGANIZACIONAIS: \\ O CAMPO DA BIOTECNOLOGIA EM ANÁLISE \\ Cleverson Renan da Cunha - Marlene Catarina de Oliveira Lopes Melo}

intercâmbio como dinheiro ou conhecimento; e a reflexividade institucional, que corresponde à entrada contínua de conhecimento afetando as ações dos indivíduos e grupos, desestabilizando certas formas básicas de relações de confiança e resultando na ampliação da sensação de instabilidade e incerteza.

A separação entre tempo e espaço tende a impedir a formação das bases tradicionais de confiança fundamentadas na família, na amizade e no parentesco. Ao mesmo tempo, os sistemas abstratos tendem a desempenhar papel semelhante ao transferir a confiança rosto no rosto para o sistema legal, o governo e os códigos de conduta, para citar alguns exemplos, fazendo surgir uma situação ambivalente. De um lado os indivíduos depositam confiança em instituições ou mecanismos técnicos avalizados pelo conhecimento científico ou pela tradição e, de outro, assumem uma atitude reservada, intimista, sem abertura para a interação com o outro em relações pessoais. A confiança é referenciada mais pelos sistemas abstratos ou peritos do que pelo especialista. Já a reflexividade social é baseada na afirmação de que as condições em que vivemos hoje na sociedade são cada vez mais o resultado de nossas próprias ações e, inversamente, nossas ações vivem cada vez mais para administrar ou enfrentar os riscos e oportunidades que nós mesmos criamos. Então, a confiança não é dada apenas pela filiação a uma família ou comunidade, mas também é construída por meio de escolhas. Assim, a separação entre tempo e espaço, os mecanismos de desencaixe e a reflexividade institucional desestimulam certas formas básicas de relações de confiança dos atributos de contextos locais.

Aulakh, Sahay e Kotabe (1996) afirmam que, embora a confiança seja propriedade de expectativa de comportamento bilateral, existente entre indivíduos, ela também pode ser estendida para troca entre organizações, uma vez que os relacionamentos interorganizacionais são gerenciados por indivíduos em cada organização. Ainda segundo esses autores, a confiança nas relações interorganizacionais inclui um conjunto de expectativas entre parceiros com relação ao comportamento dos diversos indivíduos e à satisfação de cada um.

De maneira complementar, Barney e Hansen (1994) defendem que a confiança interorganizacional depende da confiança interpessoal entre indivíduos que ligam os setores de fronteira das organizações. Isso evidencia, além dos aspectos institucionais, as relações afetivas e cognitivas que também estão presentes nos relacionamentos entre empresas e, como descrevem Zucker (1986), Zaheer, McEvily e Perrone (1998) e Giddens (1979), com o passar do tempo esses padrões do relacionamento interpessoal acabam por se institucionalizar nos relacionamentos interorganizacionais.

Na teoria da estruturação social proposta por Luhmann (1979) a confiança é definida como um mecanismo em que os atores sociais reduzem a complexidade interna do seu sistema de interação. Isto pode ocorrer pela adoção de expectativas específicas sobre o comportamento futuro de outros pela 


\section{A CONFIANÇA NOS RELACIONAMENTOS INTERORGANIZACIONAIS: \\ O CAMPO DA BIOTECNOLOGIA EM ANÁLISE \\ Cleverson Renan da Cunha - Marlene Catarina de Oliveira Lopes Melo}

seleção de possibilidades, podendo basear-se em processos históricos, em características compartilhadas ou em mecanismos institucionais. Luhmann destaca três tipos de confiança: processual, baseada em características e a institucional.

A confiança processual está ligada ao passado ou a trocas realizadas anteriormente. É um processo cumulativo e incremental de construção da confiança por meio da acumulação gradual de conhecimento direto ou indireto sobre o outro, como a reputação, a marca e a garantia de qualidade. Pressupõe um grau de estabilidade e a existência de uma baixa troca de firmas e outras instituições de mercado, sendo esse tipo de confiança deliberadamente desenvolvido pelas empresas (Luhmann, 1979). A confiança baseada em características surge da similaridade social e assume congruência social entre o que confia e o depositário da confiança, por pertencer ao mesmo grupo social ou à mesma comunidade. Compartilham uma mesma religião, status ético ou background familiar, que garante a construção de um mundo em comum. Tal confiança baseia-se na atribuição de valores e não pode ser deliberadamente criada (Luhmann, 1979).

A confiança institucional parte da consideração de que a confiança não pode ser somente gerada pela familiaridade interpessoal. Os atores organizacionais podem não possuir características pessoais em comum ou uma história que garanta trocas futuras, mas mesmo assim deve haver uma forma impessoal de confiança. A confiança institucional é formada pela estrutura social formal, em que os mecanismos legais tendem a reduzir os riscos de confiança e tornam mais fácil sua existência, podendo ser deliberadamente produzida com a consideração de que seus mecanismos necessitam ser legitimados socialmente para serem efetivos (Luhmann, 1979).

Diferentemente da escolha racional (Coleman, 1990) e da economia institucional (Williamson, 1979), os pressupostos da teoria do sistema social abordam a confiança em um contexto mais amplo, além do cálculo utilitário de consequiências. A confiança é vista, então, como a soma dos aspectos racionais e sociais.

Assim, a teoria proposta por Luhmann (1979) pode ser útil para aprofundar as idéias propostas por Ring e Van de Ven (1994), pois permite analisar os relacionamentos interorganizacionais de uma forma processual, considerando os diversos aspectos presentes no contexto em que os relacionamentos se desenvolvem, incluindo os aspectos institucionais, bem como as características dos indivíduos que participam desses relacionamentos ou os gerenciam. 


\section{A CONFIANÇA NOS RELACIONAMENTOS INTERORGANIZACIONAIS: \\ O CAMPO DA BIOTECNOLOGIA EM ANÁLISE \\ Cleverson Renan da Cunha - Marlene Catarina de Oliveira Lopes Melo}

\section{PROCEDIMENTOS METODOLÓGICOS}

Como Blomqvist (1997), entendemos que a confiança é mais a propriedade de uma unidade coletiva do que de indivíduos isolados. Isto sugere que o relacionamento - ao invés dos indivíduos - deve ser a unidade de análise na pesquisa da confiança. Devido ao estágio avançado e à importância do setor para o campo da biotecnologia, foi escolhida a subárea de saúde humana como universo do estudo. Diversas entrevistas foram realizadas com representantes de entidades relacionadas com a biotecnologia no Brasil, como a Fundação Biominas, o Pólo de Biotecnologia do Rio de Janeiro, a ABRABI (Associação Brasileira de Biotecnologia) e o SINDIBIO (Sindicato das Empresas de Biotecnologia), com a intenção de encontrar relacionamentos construídos objetivando-se a pesquisa e o desenvolvimento de novos produtos. Após as entrevistas e a análise de documentos secundários foram escolhidas três organizações que desenvolvem cooperação tecnológica para serem estudadas em profundidade, tendo como orientação os seguintes critérios: maturidade do relacionamento, acesso às informações, importância da parceria na estratégia da organização e utilização de procedimentos biotecnológicos. As entrevistas revelaram a existência de poucos relacionamentos cooperativos no campo da biotecnologia para a pesquisa e o desenvolvimento de novas tecnologias.

A seleção foi realizada por acessibilidade e por tipicidade, tendo sido escolhidos os relacionamentos que o pesquisador considerou representativos da população-alvo. Das empresas selecionadas foram ouvidos informantes responsáveis pela definição e pelo gerenciamento do envolvimento da empresa com seus parceiros, com a finalidade de descrever as relações que sua empresa mantinha com a organização-parceira, totalizando treze entrevistas. As principais fontes de informações foram as entrevistas estruturadas e semi-estruturadas, a análise bibliográfica e a análise documental de comunicações, memorandos, documentos administrativos, relatórios, artigos de jornais e revistas, buscando a convergência de várias fontes de evidência para a compreensão do fenômeno (Yin, 2001; Miles e Huberman, 1992).

Por serem os construtos de interesse normalmente estudados nas relações interorganizacionais de difícil observação, o uso de informantes-chave tem sido indicado nesse nível de análise. Esse instrumento é apropriado quando o conteúdo da pesquisa é complexo ou a informação em profundidade não pode ser conseguida em um processo de respondentes representativos. A análise dos dados foi feita de forma processual, como defendida por Miles e Huberman (1992), partindo da coleta de dados e seguida de sua apresentação, até culminar na redução dos dados, para a elaboração da conclusão, em um processo dinâmico. 


\section{A CONFIANÇA NOS RELACIONAMENTOS INTERORGANIZACIONAIS: \\ O CAMPO DA BIOTECNOLOGIA EM ANÁLISE \\ Cleverson Renan da Cunha - Marlene Catarina de Oliveira Lopes Melo}

Os dados secundários foram analisados qualitativamente com o uso da técnica de análise documental. Os dados primários também foram analisados de forma qualitativa, porém com o uso do método de análise de conteúdo das entrevistas gravadas e transcritas, em uma abordagem qualitativa (Bardin, 1977; Selltiz et al., 1987).

De acordo com Yin (2001), um dos grandes problemas do estudo de caso é sua validade externa, e o presente artigo não constitui exceção. As conclusões aqui elaboradas não são passíveis de generalização para outras empresas em razão do pequeno número de casos estudados e das diferenças existentes entre as empresas do campo. O uso de informantes é indicado pela literatura para o propósito da presente pesquisa. No entanto, corre-se o risco de não se conseguir, com os instrumentos utilizados, resgatar a complexidade das experiências vividas pelo entrevistado.

\section{APRESENTAÇÃO DOS CASOS}

Dada a limitação do escopo desse trabalho, os dados serão apresentados de forma condensada, com os resultados discrepantes em evidência. Inicialmente serão apresentadas as organizações participantes da amostra e o tipo de relacionamento interorganizacional desenvolvido, culminando na descrição e análise da confiança nos relacionamentos colaborativos entre as organizações em estudo.

A primeira empresa analisada foi a Katal Biotecnológica de Belo Horizonte. Trata-se de uma pequena empresa que atua na produção, pesquisa e desenvolvimento de kits para diagnóstico em saúde humana. Ela surgiu no início da década de 1990 como um spin-off da UFMG, mas foi somente em 1997 - com sua entrada na Incubadora de Empresas de Base Tecnológica, da Fundação Biominas - que essa empresa começou a tomar as feições atuais. Os produtos da Katal Biotecnológica são desenvolvidos com tecnologia própria e, atualmente, a empresa faz uso de duas plataformas: os diagnósticos bioquímicos e os imunodiagnósticos. A primeira é utilizada para testes mais simples, como os de glicose, creatinina e colesterol, entre outros. Os produtos resultantes são de baixo valor agregado e custo reduzido, sendo amplamente utilizados no Brasil, principalmente por laboratórios menores. A segunda plataforma visa produzir testes para detectar anticorpos, antígenos ou linfócitos para o diagnóstico de infecções, de doenças autoimunes, de processos alérgicos ou neoplásicos e também para a detecção/quantificação de hormônios ou drogas.

A Biomm S.A., segunda organização estudada, é resultante do processo de cisão da Biobrás em 2001, cuja estrutura industrial e a marca foram vendidas para uma empresa farmacêutica dinamarquesa 


\section{A CONFIANÇA NOS RELACIONAMENTOS INTERORGANIZACIONAIS: \\ O CAMPO DA BIOTECNOLOGIA EM ANÁLISE \\ Cleverson Renan da Cunha - Marlene Catarina de Oliveira Lopes Melo}

por U\$ 31,7 milhões, e os laboratórios e a gestão da patente da insulina recombinante ficaram com a Biomm S.A. Atualmente, ela oferece dois itens de serviços: a plataforma tecnológica de produção de proteínas recombinantes e a tecnologia da vacina contra a leishmaniose. A empresa realiza três tipos de parcerias: para o desenvolvimento tecnológico, para transferência de tecnologia e com agentes que trabalhavam para a Biobrás em diversas partes do mundo. Das parcerias citadas, a primeira é a mais importante. Ela é construída principalmente com base em relacionamentos interpessoais, nos quais o conhecimento e a reputação são pontos fortes. Esse tipo de rede não se encontra definida em um papel - é virtual -, sendo construída e reconstruída ao longo do tempo à medida que surgem os diversos interesses.

O terceiro caso é do Instituto de Tecnologia de Imunobiológicos, o Bio-Manguinhos. Trata-se de uma unidade da Fiocruz (Fundação Oswaldo Cruz) dedicada ao desenvolvimento e produção de vacinas, reagentes e kits diagnósticos, com sede na cidade do Rio de Janeiro e ligado ao Ministério da Saúde. O principal motivo que tem levado a organização a buscar acordos é a necessidade de dominar técnicas de produção que levariam muito tempo para a sua maturação. Nos últimos anos, o Brasil não desenvolveu tecnologia para a produção de vacinas, explorando somente as poucas que dominava. No entanto, recentemente o Ministério da Saúde mudou sua política imunológica, exigindo do BioManguinhos e de outras organizações rápida adequação aos novos padrões. Como o desenvolvimento é demorado, em alguns casos chegando a mais de 15 anos, tem-se optado pela compra de tecnologia ou pelo desenvolvimento cooperado para acelerar o atendimento às demandas do Ministério.

\section{ANÁLISE DOS CASOS}

Entende-se que a confiança - assim como outras questões ligadas ao relacionamento interpessoal e interorganizacional, como contrato, poder e risco - está inserida em um contexto cultural, social, econômico e político mais amplo, exercendo influências e sendo alvo delas. No caso das empresas analisadas, esses impactos são percebidos tanto pelas bases que sustentam a confiança como pela forma de gerenciar o relacionamento.

Nas três empresas pesquisadas foram identificadas algumas parcerias, com destaque para as comerciais e as de desenvolvimento tecnológico. Nesses casos, as alianças para pesquisa e desenvolvimento constituem mais do que uma decisão estratégica, pois envolvem a própria 


\section{A CONFIANÇA NOS RELACIONAMENTOS INTERORGANIZACIONAIS: \\ O CAMPO DA BIOTECNOLOGIA EM ANÁLISE \\ Cleverson Renan da Cunha - Marlene Catarina de Oliveira Lopes Melo}

sobrevivência organizacional no futuro, corroborando as idéias de Aldrich (1979) da auto-sustentação.

Além de um desejo, as parcerias têm surgido como uma estratégia para a adequação às necessidades dos diversos envolvidos. Se, por um lado, elas têm sido imprescindíveis, por outro, as organizações ainda não possuem mecanismos que garantam a eficácia desses relacionamentos.

Nesses casos, a cooperação é o mote maior, estando presentes fortes instrumentos formais e informais de comunicação e controle e, na maioria dos casos, não se tratando de redes interorganizacionais de pesquisa, e sim de relações diádicas com propósitos bem delineados, seja por contratos ou por acordos verbais. Na prática, existem redes de pessoas, uma vez que diversas trocas acontecem entre os pesquisadores dessas empresas, sejam orientadas por relações de amizade, seja por participarem de um outro grupo de pesquisa, configurando uma rede dinâmica (Miles e Snow, 1992). Assim, os contatos mantidos com uma dada organização em uma relação diádica transcendem seus limites, pois cada uma dessas organizações também faz parte de outras alianças e redes interpessoais e interorganizacionais.

A terceira instituição analisada constituiu um caso particular. Por fazer parte de um grupo maior, a Fiocruz, e por ser um dos seus braços operativos, o Instituto Bio-Manguinhos possui diversas interações com outras unidades da Fundação que se assemelham à metáfora da rede. Além das contribuições formais realizadas entre as unidades, diversos elos são criados e alimentados pelos pesquisadores, técnicos e pessoal de suporte, em alguns casos sendo de difícil identificação o que é o Bio-Manguinhos ou a Fiocruz.

Os principais problemas decorrentes do desenvolvimento tecnológico cooperado destacados pelas empresas se concentram na dificuldade de gerenciar o conhecimento inerente às atividades de pesquisa e no cumprimento de prazos. Muitas vezes não é possível determinar os prazos a priori, como, por exemplo, no caso de uma pesquisa que necessita do resultado de uma cultura inédita de microorganismos feita no laboratório. Como não existem outros parâmetros para sua comparação, fica impossível estabelecer o tempo de conclusão da pesquisa de forma adequada. Em muitos casos esses tempos são estimados, mas, na prática, não podem ser cumpridos por motivos alheios à vontade dos pesquisadores.

Quanto aos instrumentos para resolução de problemas, em todos os casos, a negociação e o diálogo foram indicados como os principais. Isso pode ser explicado por duas vertentes: a primeira é que em nossa sociedade não é bem visto o embate frontal de idéias, muito menos de desavenças (DaMatta, 1997), resultado de nossas heranças históricas. O interesse em continuar o relacionamento, o segundo caminho, parece ser ainda mais importante no contexto da biotecnologia, uma vez que os 


\section{A CONFIANÇA NOS RELACIONAMENTOS INTERORGANIZACIONAIS: \\ O CAMPO DA BIOTECNOLOGIA EM ANÁLISE \\ Cleverson Renan da Cunha - Marlene Catarina de Oliveira Lopes Melo}

relacionamentos para conseguir os resultados esperados necessitam de grande tempo de maturação. No Bio-Manguinhos, além desse caminho, a resolução via sistema legal também é adotada, pois em muitos casos a negociação não é permitida pela legislação brasileira. Nos relacionamentos mantidos pela organização, o contrato é tido como um trilho, e não um caminho. Dessa forma, o acordo formal não é criado ou usado com possibilidades de adaptações e o seu não-cumprimento obriga o lesado a buscar outras formas de resolução que não a negociação, funcionando como entrave para o desenvolvimento de relações de confiança.

A motivação principal para a formação dos relacionamentos é a complementação técnica. Esta é vista como natural, uma vez que sua busca é necessária em atividades que envolvem conhecimentos aprofundados e variados, como a produção de medicamentos, seja para reduzir prazos de lançamento de produtos, seja pela incapacidade de domínio de tantas técnicas.

Tanto a Biomm quanto o Bio-Manguinhos têm parte significativa dos seus resultados na área de desenvolvimento tecnológico, como conseqüência de parcerias, o que evidencia a importância desses relacionamentos para a empresa. Já para a Katal, as parcerias visando ao desenvolvimento é algo recente, mas que já vem apresentando resultados expressivos, que culminaram inclusive com o pedido de uma patente internacional. Foi possível perceber nas entrevistas que todas as empresas continuam pensando nas parcerias como instrumentos para inovação nos próximos anos.

Em relação à confiança, as respostas obtidas com as entrevistas e a análise documental evidenciam a existência de um padrão de entendimento sobre o que é a confiança, seu papel na formação e desenvolvimento dos relacionamentos interorganizacionais cooperativos, assim como sua ligação com o contrato. Essa congruência também foi percebida ao comparar as respostas dos informantes na Biomm e no Bio-Manguinhos. Na Katal, em razão do seu porte e também da centralização da coordenação tanto dos relacionamentos interorganizacionais como intraorganizacionais na figura do seu diretor, o processo interno de institucionalização é mais limitado, uma vez que as outras pessoas participam pouco dessa construção.

A base da confiança de maior impacto nos relacionamentos interorganizacionais cooperativos para pesquisa e desenvolvimento nas empresas pesquisadas foi a processual e, nes se contexto, destacam-se as trocas passadas, a reputação, a qualidade de produtos e serviços, entre outros. Para os informantes, quanto mais esses itens estiverem diretamente relacionados ao objeto da aliança, maior será sua capacidade de influenciar no desenvolvimento da confiança, mas, de uma forma geral, o resultado de todos os tipos de trocas tende a ser considerado ao se pensar em outros tipos de transação. Os casos reforçam as idéias de Luhmann (1979) de que esse tipo de confiança pode ser 


\section{A CONFIANÇA NOS RELACIONAMENTOS INTERORGANIZACIONAIS: \\ O CAMPO DA BIOTECNOLOGIA EM ANÁLISE \\ Cleverson Renan da Cunha - Marlene Catarina de Oliveira Lopes Melo}

deliberadamente desenvolvido pelas empresas por meio de experiências em relacionamentos com riscos crescentes, cuidados especiais com a reputação, participação em outras atividades cooperadas e programas de controle interno da produção e pesquisa.

Ao se analisar o processo de formação e manutenção dos relacionamentos interorganizacionais cooperativos, percebe-se que cada base da confiança tende a desempenhar um papel distinto e importante. Quando se trata de acordos entre empresas que não se conhecem bem, o contrato tem sido usado como um instrumento para diminuição de riscos, permeado pelo cálculo utilitário de conseqüências. Nesses relacionamentos, a confiança tende a desempenhar papel secundário, pois ela ainda não surgiu ou foi criada. De acordo com a análise dos casos, percebe-se que, nessas situações, pela falta da base processual, as outras bases têm sido adotadas como referência. Mas, se o relacionamento atual é reflexo ou continuidade de outros, isso tende a se reverter, diminuindo a necessidade de contratos ou a quantidade de cláusulas formais.

Se já existe um sentimento de confiança entre os participantes da aliança no momento inicial do relacionamento, a parceria tende a se desenvolver de forma mais rápida. Assim aumenta a troca de informações, além de facilitar a resolução de problemas, evidenciando a importância de se participar de redes e outros tipos de parceria mesmo que seja só para se tornar conhecido ou descobrir parceiros para novos empreendimentos cooperados.

Segundo os informantes, para se chegar à fase de negociação já se faz necessária a existência de algum nível de confiança entre os parceiros, pois, caso contrário, o relacionamento tende a nem existir. Quanto mais bases estiverem presentes nessa relação, maior tende a ser a segurança para utilizar a confiança como instrumento para a redução de riscos em situações futuras. Percebeutse que, para cada parceiro e tipo de questão a ser negociada, as bases da confiança podem ser alteradas, mas em todos os casos a processual foi a mais citada. Quando se trata de questões facilmente identificadas e quantificáveis ou com alto nível de risco, a confiança tende a ceder lugar ao contrato como instrumento para a condução do relacionamento.

A confiança tende a acelerar a execução do desenvolvimento tecnológico, realizada por meio de pesquisas práticas, testes e simulações, assim como a diminuir os custos de controle e inspeção, independentemente de sua origem. Nessa fase, além da confiança, inspeções nos laboratórios e apresentações de resultados parciais consistentes tendem a aprimorar o sentimento de confiabilidade no parceiro, realimentando constantemente esse relacionamento, ao mesmo tempo em que diminuem os controles formais. Essa fase foi destacada como a mais importante para a continuidade dos 


\section{A CONFIANÇA NOS RELACIONAMENTOS INTERORGANIZACIONAIS: O CAMPO DA BIOTECNOLOGIA EM ANÁLISE \\ Cleverson Renan da Cunha - Marlene Catarina de Oliveira Lopes Melo}

relaciona mentos e também como um momento para testar o nível de confiança no parceiro. Mais uma vez, as bases processuais foram identificadas como as mais importantes.

Na tomada de decisão, a confiança está interligada com a possibilidade de desistir de certas vantagens ou exigências em detrimento de ganhos futuros. A confiança tende a oferecer mais segurança sobre o comportamento do parceiro e, conseqüentemente, facilita a aceitação ou convivência com possíveis problemas decorrentes da interação. Nesse momento, se ela estiver calcada em características, as decisões tendem a ser mais rápidas e seguras, pois se compartilham mais as informações.

Em cada etapa do processo, a confiança é tanto um desafio a ser vencido, pois sempre existe a possibilidade do rompimento do acordo preestabelecido, como um instrumento para o estreitamento das relações, tendo em seu seio oportunidades e desafios conflitantes (veja Quadro 2). O equilíbrio entre a flexibilidade da confiança e as garantias oferecidas pelo contrato parece ser um objetivo que está constantemente sendo perseguido pelas empresas e gestores em análise. 


\section{A CONFIANÇA NOS RELACIONAMENTOS INTERORGANIZACIONAIS: \\ O CAMPO DA BIOTECNOLOGIA EM ANÁLISE \\ Cleverson Renan da Cunha - Marlene Catarina de Oliveira Lopes Melo}

\section{QUADRO 2 - Desafios e oportunidades da confiança nos relacionamentos interorganizacionais pesquisados.}

\begin{tabular}{|c|c|c|c|}
\hline & KATAL & BIOMM & BIO-MANGUINHOS \\
\hline \multirow{3}{*}{ 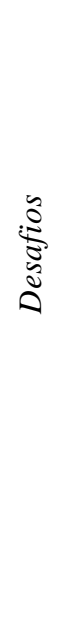 } & $\begin{array}{l}\text { Desenvolvimento de } \\
\text { relac ionamentos que } \\
\text { transcendem as relações } \\
\text { pessoais. }\end{array}$ & $\begin{array}{l}\text { O desenvolvimento da confiança } \\
\text { é dificultado pela natureza } \\
\text { intangível do negócio da } \\
\text { empresa. }\end{array}$ & $\begin{array}{l}\text { Equilíbrio entre os mecanismos } \\
\text { formais de controle e o } \\
\text { desenvolvimento da confiança } \\
\text { nos parceiros. }\end{array}$ \\
\hline & $\begin{array}{l}\text { Institucionalização dos } \\
\text { mecanismos de coordenação dos } \\
\text { relacionamentos. }\end{array}$ & $\begin{array}{l}\text { Necessidade de continuar } \\
\text { inovando para participar de } \\
\text { novas redes de pesquisa. }\end{array}$ & $\begin{array}{l}\text { Conciliar os mecanismos de } \\
\text { controle formal com um } \\
\text { ambiente propício para a } \\
\text { confiança. }\end{array}$ \\
\hline & $\begin{array}{l}\text { Desenvolver a confiança em um } \\
\text { ambiente hostil à continuidade e } \\
\text { à manutenção dos acordos } \\
\text { informais, como é o caso dos } \\
\text { relacionamentos com as } \\
\text { universidades. }\end{array}$ & $\begin{array}{l}\text { Manutenção do histórico de } \\
\text { sucesso da Biomm para } \\
\text { continuar usufruindo de sua } \\
\text { reputação. }\end{array}$ & $\begin{array}{l}\text { Desenvolver mecanismos } \\
\text { institucionais (valores e normas } \\
\text { sociais estáveis) que favoreçam } \\
\text { o desenvolvimento da confiança. }\end{array}$ \\
\hline \multirow{3}{*}{ 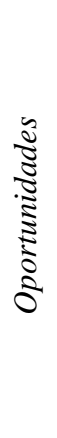 } & Empresa inovadora. & $\begin{array}{l}\text { Tradição no desenvolvimento de } \\
\text { pesquisa cooperada. }\end{array}$ & $\begin{array}{l}\text { Tradição no desenvolvimento de } \\
\text { pesquisa cooperada. }\end{array}$ \\
\hline & $\begin{array}{l}\text { A centralização das decisões } \\
\text { facilita a realização de acordos e } \\
\text { a tomada de decisões. }\end{array}$ & $\begin{array}{l}\text { Grupo executivo coeso com } \\
\text { objetivos e visão bem definidos. }\end{array}$ & $\begin{array}{l}\text { Corpo técnico altamente } \\
\text { capacitado e reconhecido } \\
\text { internacionalmente. }\end{array}$ \\
\hline & $\begin{array}{l}\text { Reputação de empresa enxuta e } \\
\text { que possui capacidade de } \\
\text { transformar pesquisa em } \\
\text { produto. }\end{array}$ & $\begin{array}{l}\text { Reputação de empresa de } \\
\text { resultados, influenciando } \\
\text { positivamente a confiança de } \\
\text { seus parceiros. }\end{array}$ & $\begin{array}{l}\text { Grande reputação no mercado } \\
\text { como instituição séria, que afeta } \\
\text { positivamente a confiança de } \\
\text { seus parceiros. }\end{array}$ \\
\hline
\end{tabular}

É possível notar a transição das relações baseadas na confiança para aquelas orientadas pelo contrato e outros mecanismos institucionais de gerenciamento. Não que a confiança deixe de existir, mas ela vem passando de papel central para complementar aos outros instrumentos. Nesse caso, o contrato tem sido usado não por sua capacidade de garantir que os objetivos sejam atendidos, mas sim como adequação mimética ao que acontece em outras realidades, como a norte-americana, por exemplo, porém sem a existência de instituições que dão respaldo à execução dos contratos, como nos Estados Unidos.

Nos casos analisados ficou evidente que a confiança é uma questão complexa e está no âmago da formação e desenvolvimento de acordos cooperativos. Se, por um lado, ela é condição sine qua non para a existência de um relacionamento, por outro, ela sozinha não consegue oferecer condições 


\section{A CONFIANÇA NOS RELACIONAMENTOS INTERORGANIZACIONAIS: \\ O CAMPO DA BIOTECNOLOGIA EM ANÁLISE \\ Cleverson Renan da Cunha - Marlene Catarina de Oliveira Lopes Melo}

suficientes para a consecução dos objetivos dessas parcerias. Pela análise da Katal, da Biomm e do BioManguinhos verificoutse a importância de se considerar a confiança e outros aspectos socialmente construídos na tentativa de entender a criação de relacionamentos mais eficazes para as organizações envolvidas.

Assim, o desenvolvimento de relacionamentos interorganizacionais mais efetivos passa obrigatoriamente em primeiro lugar pelo aprimoramento dos sistemas de gestão, envolvendo planejamento de longo prazo, confiança nas relações intra-organizacionais, mecanismos de controle e coordenação mais sofisticados, como também pela solidificação de relações sociais como associações, sindicatos e fóruns de discussão e, finalmente, também envolve o aprimoramento dos sistemas de proteção, como a justiça e a legislação.

\section{CONCLUSÃO}

No contexto organizacional contemporâneo as relações cooperativas vêm sendo adotadas pelas organizações como forma de maximizar a consecução de seus objetivos. Nesses casos, a confiança tem sido indicada como necessária para a realização de alianças eficazes, principalmente em campos organizacionais dinâmicos como o da biotecnologia. Objetivourse com o presente trabalho identificar a influência da confiança no processo de formação e desenvolvimento de relações interorganizacionais cooperativas em empresas que fazem uso de biotecnologias em saúde humana. Para atingir esse propósito, foram realizados estudos de casos em três empresas brasileiras que possuem desenvolvimento tecnológico cooperado, buscando responder ao problema de pesquisa proposto.

Percebeu-se que para as organizações que fazem uso de biotecnologias, questões como amizade, parentesco e outras relações primárias não são mais suficientes para a formação e o desenvolvimento de relacionamentos nesse campo organizacional. No entanto, outros padrões sustentados pela sociedade civil, pelo Estado e por suas instituições ainda não oferecem todas as condições necessárias para a adoção de novas bases de confiança. Aqui cabe destacar a ineficiência do judiciário que leva as organizações a celebrarem contratos e outros mecanismos de controle mais como mimetismo. Utilizam-se também a confiança em pessoas e instituições mediadoras no sentido de diminuir os riscos do relacionamento.

Na literatura internacional utilizada como referência teórica para o presente estudo, a existência da confiança interorganizacional é comum nos relacionamentos entre entidades autônomas distintas. 


\section{A CONFIANÇA NOS RELACIONAMENTOS INTERORGANIZACIONAIS: \\ O CAMPO DA BIOTECNOLOGIA EM ANÁLISE \\ Cleverson Renan da Cunha - Marlene Catarina de Oliveira Lopes Melo}

No entanto, isso não parece tão claro nas nossas relações, pois em muitos casos foi evidenciado que isso não é possível, pois, segundo os entrevistados, não se confia em organizações e sim em pessoas.

As atividades necessárias ao aprimoramento das biotecnologias necessitam de interações que transcendem o departamento de pesquisa, a organização, seu setor de atuação e até países. Nesse contexto, os sistemas de desencaixe são necessários para conectar as práticas locais às relações sociais globalizadas, fazendo uma transição entre os compromissos com rostos e os compromissos sem rostos dos sistemas abstratos (Giddens, 1991), baseados em sistemas peritos como a Justiça ou a Organização Mundial do Comércio (OMC).

Nesse caso, existiriam duas espécies de confiança: a estabelecida entre indivíduos que se conhecem bem, baseadas em um relacionamento de longo prazo, com trocas de risco crescentes decorrentes do comportamento confiável, e a confiança relativa aos mecanismos de desencaixe, formados principalmente pelos sistemas peritos, que em nosso meio carecem de aprimoramento.

Nas empresas estudadas, os primeiros relacionamentos para desenvolvimento tecnológico cooperado surgiram das ligações pessoais como amizade e parentesco. No entanto, graças à elevação do nível da tecnologia envolvida com a biotecnologia, essa base já não é tão importante para a confiança interorganizacional. Nos relacionamentos mais recentes a confiança intermediada por uma terceira pessoa ou organização e o contrato vêm recebendo atenção. Mas esse último ainda é usado de forma limitada, mais como um mecanismo de salvaguarda do que como um instrumento efetivo para o gerenciamento dos relacionamentos. Em muitos casos isto se deve à ineficiência do sistema jurídico/legal brasileiro, além das dificuldades inerentes ao desenvolvimento de tecnologias que fazem uso de processos biotecnológicos.

Se, por um lado, as bases tradicionais não conseguem sustentar a confiança nos relacionamentos do campo em estudo, por outro, os mecanismos socialmente institucionalizados não oferecem ainda respostas satisfatórias às necessidades das empresas atuantes no campo da biotecnologia no Brasil.

Ainda somos uma sociedade tradicional, marcada pela informalidade, por relações baseadas em características e afinidades, mas nossas empresas começam a aperfeiçoar padrões racionais em seus relacionamentos, embora ainda não possuamos aspectos institucionais desenvolvidos para dar-lhes suporte. No campo da biotecnologia isso é ainda mais evidente, porque as atividades a ele relacionadas não são suficientemente suportadas por laços de amizade e parentesco, porém ainda não possuímos um sistema legal que possibilite nossa interação em um sistema abstrato (Giddens, 1991).

Para as empresas que fazem uso das biotecnologias, a pouca institucionalização dos mecanismos abstratos que dão suporte ao desenvolvimento de relações entre os membros da sociedade 


\section{A CONFIANÇA NOS RELACIONAMENTOS INTERORGANIZACIONAIS: \\ O CAMPO DA BIOTECNOLOGIA EM ANÁLISE \\ Cleverson Renan da Cunha - Marlene Catarina de Oliveira Lopes Melo}

levam essas organizações a desenvolverem estratégias alternativas para lidar com a confiança, além dos outros aspectos presentes nos relacionamentos.

Diante das conclusões anteriores, das perguntas da pesquisa e da impossibilidade de analisar toda a complexidade que envolve a confiança nos relacionamentos, propõem se como sugestões de pesquisas futuras: (a) pesquisas comparativas acerca da influência da confiança em diferentes campos organizacionais, analisando a percepção de todos os envolvidos, desde as relações diádicas às redes de pesquisa e desenvolvimento; e (b) a utilização de outras metodologias para o entendimento da confiança, com destaque para estudos longitudinais e pesquisas de cunho antropológico.

\section{REFERÊNCIAS}

ACHROL, R.; SCHEER, K.; STERN, L. W. Designing successful transorganizational marketing alliances. Marketing Science Institute Working Paper n. 90118. Cambridge, MA: Marketing Science Institute, 1990.

ALDRICH, H. Organizations \& environments. Englewood Cliffs: Prentice-Hall, 1979.

ALTER, C.; HAGE, J. Organizations working together. Newbury Park, CA: Sage, 1993.

ARGYLE, G. W. Cooperation: the basis of sociability. London: Rutledge, 1991.

AULAKH, P. S.; KOTABE, M.; SAHAY, A. Trust and Performance in Cross-border Marketing Partnerships: a behavioral approach. Journal of International Business Studies, v. 27, n. 5, p. 1005$1032,1996$.

BACHMANN, R. et al. Trust and control in organizational relations. Organization Studies, v. 22, n. 2, p. 337-365, mar. 2001.

BARDIN, L. Análise de conteúdo. Lisboa: Edições 70, 1977. 


\section{A CONFIANÇA NOS RELACIONAMENTOS INTERORGANIZACIONAIS: O CAMPO DA BIOTECNOLOGIA EM ANÁLISE \\ Cleverson Renan da Cunha - Marlene Catarina de Oliveira Lopes Melo}

BARNEY, J. B.; HANSEN, M. H. Trustworthiness as a source of competitive advantage. Strategic Management Journal, v. 15, special issue, p. 175-90, 1994.

BLOMQVIST, B. The many faces of trust. Scandinavian Journal of Management, v. 13, n. 3, p. 271286, 1997.

CARVAlHO, A. P. C. Biotecnologia no Brasil e no Reino Unido: Possibilidades de Cooperação. Disponível em:<http://www.mre.gov.br>. Acessado em: 03 mar. 2004.

COLEMAN, J. S. The foundations of social theory. Cambridge, MA: Harvard University Press, 1990.

DAMATTA, R. Carnavais, malandros e heróis: para uma sociologia do dilema brasileiro. Rio de Janeiro: Rocco, 1997.

DIMAGGIO, P. J.; POWELL, W. W. The iron cage revisited: institutional isomorphism and collective rationality in organizational fields. American Sociological Review, v. 48, n. 2, p. 31-41, 1983.

DODGSON, M. Learning, trust and technological collaboration. Human relations, v. 46, n. 1, p. 77-95, 1993.

DOZ, Y. L; HAMEL, G. A vantagem das alianças: a arte de criar valor através de parcerias. São Paulo: Qualitymark, 2000.

FUKUYAMA, F. Trust: the social virtues and the creation of prosperity, London: Hamish Hamilton, 1995.

GIDDENS, A. As conseqüências da modernidade. São Paulo: Unesp, 1991.

GIDDENS, Anthony. Central problems in social theory: action, structure, and contradiction in social analysis. Berkeley, CA: University of California Press, 1979. 


\section{A CONFIANÇA NOS RELACIONAMENTOS INTERORGANIZACIONAIS: O CAMPO DA BIOTECNOLOGIA EM ANÁLISE \\ Cleverson Renan da Cunha - Marlene Catarina de Oliveira Lopes Melo}

GRANDORI, A.; SODA, G. Inter-firm networks: antecedents, mechanisms and forms. Organizational Studies, v. 16, n. 2, p. 183-214, 1995.

LANE, C. Introduction: theories and issues in the study of trust. In: LANE, C.; BACHMANN, R. (Ed.) Trust within and between organizations: conceptual issues and empirical applications. Oxford: Oxford University Press, 1998.

LUHMANN, N. Trust and power. Chichester: John Wiley, 1979.

MILES, M. B.; HUBERMAN, A. M. Qualitative data analysis: an expanded sourcebook. London: Sage, 1994.

MILES, R. E.; SNOW, C. C. Cases of failure in network organizations. California Management Review, v. 34, n. 4, p. 53-72, 1992.

NEWELL, S. SWAN, J. Trust and inter-organizational networking. Human Relations, v. 53, n. 10, p. 1287-1328, 2000.

OLIVER, A. L. Networking network studies: an analysis of conceptual configurations in the study of inter-organizational relationships. Organization Studies, v. 19, n. 4, p. 549-583, 1998.

OLIVER, C. Determinants of interorganizational relationships: integration and future directions. Academy of Management Review. v. 15. n. 2, p. 241-265, 1990.

POWELL, W. W. The social construction of an organizational field. The case of biotechnology. International Journal of Biotechnology, v. 1, n. 1, p. 42-66, 1999.

POWELL, W. W.; KOPUT, K. W.; SMITH-DOERR, L. Interorganizational collaboration and the locus of innovation: Networks of learning in biotechnology. Administrative Science Quarterly, v. 41, $\mathrm{n}$. 1, p. 116-145, 1996. 


\section{A CONFIANÇA NOS RELACIONAMENTOS INTERORGANIZACIONAIS: O CAMPO DA BIOTECNOLOGIA EM ANÁLISE \\ Cleverson Renan da Cunha - Marlene Catarina de Oliveira Lopes Melo}

RING, P. S.; VAN de VEN, A. H. Developmental process of cooperative interorganizational relationships. Academy of Management Review, v. 19, n. 1, p. 90-118, 1994.

RING, P.S.; VAN de VEN, A. H. Structuring cooperative relationships between organizations. Strategic Management Journal, v. 13, n. 17, p. 483-98, 1992.

SELLTIZ, C. et al. Metodologia de pesquisa nas relações sociais. São Paulo: EPU, 1987.

STUART, T. E. Network positions and propensities to collaborate: an investigation of strategic alliance formation in a high-technology industry. Administrative Science Quarterly, v. 43, n. 3, p. 668-698, 1998.

TRIGUEIRO, M. G. S. O clone de Prometeu: a biotecnologia no Brasil - uma abordagem para a avaliação. Brasília: UNB, 2002.

WILLIAMSON, O. E. Transaction-cost economics: the governance of contractual relations. Journal of Law and Economics, v. 22, n. 2, p. 233-61, 1979.

YIN, R. K. Estudo de caso: planejamento e métodos. Porto Alegre: Bookman, 2001.

ZAHEER, A.; McEVILY, B.; PERRONE, V. Does Trust Matter? Exploring the Effects of Interorganizational and Interpersonal Trust on Performance. Organization Science, v. 9, n. 2, p. 141$159,1998$.

ZUCKER, L. G. Production of trust: institutional sources of economic structure: 1840-1920. In: STAW, B. M.; CUMMINGS, B. M. (Eds.). Research in organizational behavior. Greenwich: JAI, 1986. p. 53-111.

Artigo recebido em 17.12.2004. Aprovado em 27.04.2006. 


\section{A CONFIANÇA NOS RELACIONAMENTOS INTERORGANIZACIONAIS: \\ O CAMPO DA BIOTECNOLOGIA EM ANÁLISE \\ Cleverson Renan da Cunha - Marlene Catarina de Oliveira Lopes Melo}

\section{Cleverson Renan da Cunha}

Doutor em Administração pela Universidade Federal de Minas Gerais. Professor do Programa de Pósgraduação da Universidade Federal do Paraná.

Interesses de pesquisa nas áreas de relações interorganizacionais, metodologia de pesquisa e estudos organizacionais.

E-mail: cleverson.cunha@gmail.com

Endereço: Rua Des. Pedro Silva, 2100. Ap. 205 Bloco B, Florianópolis - SC, 88080-700.

\section{Marlene Catarina de Oliveira Lopes Melo}

Professora Titular aposentada do Cepead/CAD/UFMG e Vice-diretora da Faculdade Novos Horizontes. Doutora em Ciências das Organizações pela Université Paris IX, Dalphin (France).

Interesses de pesquisa nas áreas de relações de poder e transformações dos sistemas de relações profissionais e de trabalho, organização do processo de trabalho e inovação tecnológica, condições de trabalho e saúde do trabalhador, dinâmica da função gerencial

E-mail: lenemelo@unihorizontes.br

Endereço: Rua Alvarenga Peixoto, n. 1270, Belo Horizonte - MG, 30180-121. 